Article

\title{
Exploring the Association between Urine Caffeine Metabolites and Urine Flow Rate: A Cross-Sectional Study
}

\author{
Shou En Wu ${ }^{1,2}$ and Wei-Liang Chen ${ }^{1,2,3, *(D)}$ \\ 1 Division of Family Medicine, Department of Family and Community Medicine, Tri-Service General Hospital \\ and School of Medicine, National Defense Medical Center, Taipei 114, Taiwan; grace830115@gmail.com \\ 2 Division of Geriatric Medicine, Department of Family and Community Medicine, Tri-Service General \\ Hospital, and School of Medicine, National Defense Medical Center, Taipei 114, Taiwan \\ 3 Department of Biochemistry, National Defense Medical Center, Taipei 114, Taiwan \\ * Correspondence: weiliang0508@gmail.com; Tel.: +886-2-8792-3311 (ext. 16567); Fax: +886-2-8792-7057
}

Received: 6 August 2020; Accepted: 9 September 2020; Published: 13 September 2020

\begin{abstract}
Examination of urine excretion of caffeine metabolites has been a simple but common way to determine the metabolism and effect of caffeine, but the relationship between urinary metabolites and urine flow rate is less discussed. To explore the association between urinary caffeine metabolite levels and urine flow rate, 1571 participants from the National Health and Nutrition Examination Survey (NHANES) 2011-2012 were enrolled in this study. We examined the association between urinary caffeine metabolites and urine flow rate with linear regression models. Separate models were constructed for males and females and for participants aged $<60$ and $\geq 60$ years old. A positive association was found between concentrations of several urinary caffeine metabolites and urine flow rate. Three main metabolites, namely, paraxanthine, theobromine, and caffeine, showed significance across all subgroups. The number of caffeine metabolites that revealed flow-dependency was greater in males than in females and was also greater in the young than in the elderly. Nevertheless, the general weakness of NHANES data, a cross-sectional study, is that the collection is made at one single time point rather than a long-term study. In summary, urinary concentrations of several caffeine metabolites showed a positive relationship with the urine flow rate. The trend is more noticeable in males and in young subgroups.
\end{abstract}

Keywords: urine caffeine metabolites; urine flow rate

\section{Introduction}

Caffeine is a common psychoactive stimulant that can be found in daily beverages such as coffee, tea, and cocoa. Its impact on various aspects has aroused the interest of researchers from different fields. In the field of physiology, the impact of caffeine on the central nervous system and peripheral organs has been widely discussed [1]. The most noticeable one is the antagonism of adenosine receptors A1 and A2 [2], which play a famous role in arousal, vigilance, and anxiety [3]. In regard to neurotransmitters, caffeine seems to affect norepinephrine, dopamine, and serotonin, which contribute to alertness [4]. In the cardiovascular system, caffeine increases heart rate and affects blood pressure, cardiac rhythm, and various cardiac diseases [5,6]. In kidneys, caffeine induces diuresis and natriuresis [7]. In the field of psychology, sleep disturbance, learning and memory, addiction, and withdrawal are popular topics related to caffeine [8-10].

In the field of pharmacology, enzyme assays, including CYP1A2, N-acetyltransferase, and xanthine oxidase, utilize caffeine and its urinary metabolites as the means of evaluation [11,12]. There are various ways of evaluating caffeine metabolism. Urine levels, serum levels, and metabolite ratios of 
caffeine act as biomarkers for diseases [13], targets for drugs [14], and probes for enzyme activity [15]. Factors that may confound the results of examinations should be taken into account when interpreting related data. Urine flow rate is undoubtedly a crucial factor when interpreting data regarding urinary caffeine metabolism, and thus the association between urinary caffeine metabolite concentrations and urine flow rate deserves attention. Previous literature discussing flow-dependency put more focus on theophylline, one of the caffeine metabolites that is well known for its therapeutic effects on asthma and chronic obstructive pulmonary disease (COPD) [16-18]. However, comprehensive studies about other caffeine metabolites are lacking. Therefore, the purpose of our study is to investigate the relationship between 14 main urinary caffeine metabolites and the urine flow rate.

\section{Materials and Methods}

\subsection{Design and Participants}

The NHANES study, a nationally representative study of population in the United States, is a cross-sectional survey based on a national sample of randomly-selected residents in the USA. It is administered by the National Center for Health Statistics (NCHS) of the Centers for Disease Control and Prevention (CDC). The survey combines three main parts. Initial screening questions determine qualified participants. Afterward, an extensive interview is held at home, which includes information such as age, gender, race, medical history, and health status. Further physical examination or clinical evaluations are performed at specially designed mobile examination centers (MECs). All interviewers received training programs and met the required standards. NHANES started in 1999 and remains a continuous annual survey, with data released every 2 years. Detailed questionnaire instruments, procedure manuals, brochures, and consent documents for the 2011-2012 NHANES are described on the NHANES website. This study gained Institutional Review Board (IRB; project identification code protocol \#2011-17) approval by the National Center for Health Statistics (NCHS) in line with the revised Helsinki Declaration. Informed consents were collected from all research participants before the data-gathering procedure and examinations were carried out.

There were 9756 participants in the NHANES dataset from 2011-2012. Data from 2009-2010 also performed urinary caffeine analysis but was abandoned due to the instrument used not being suitable for analyzing both positive and negative ions simultaneously, so each urine specimen was analyzed twice. Data from 2011-2012 was from an improved instrument so that the measurement of each specimen was done in a single analysis. After excluding those under 18 years old and those with missing data such as urine flow rate, urinary caffeine analysis, and those taking medication of benign prostatic hyperplasia and diuretics, 1410 eligible participants were involved in our analysis. Figure 1 shows a scheme of the flow chart of participant recruitment. We performed our analyses in three stages: categorizing participants as a whole population, by gender (male and female), and by age (cutoff value set at 60 years old to refer to the elderly population $[19,20]$ ).

\subsection{Measurement of Caffeine Metabolites in Urine}

Spot urine samples were collected by experienced operators at the MECs. Recorded documents included the date and time of sampling and the volume of urine collection. Samples were stored at $\leq-70{ }^{\circ} \mathrm{C}$ based on the Laboratory Procedures Manual before transportation to the National Center for Environmental Health (Centers for Disease Control and Prevention, Atlanta, GA, USA) for testing. Urinary metabolite quantification was determined by ultra-high performance liquid chromatography-electrospray ionization-tandem quadrupole mass spectrometry (UHPLC-ESI-MS/MS) (Agilent Technologies, Palo Alta, CA, USA) with stable isotope-labeled internal standards. More detailed methods are reported on the NHANES website. 


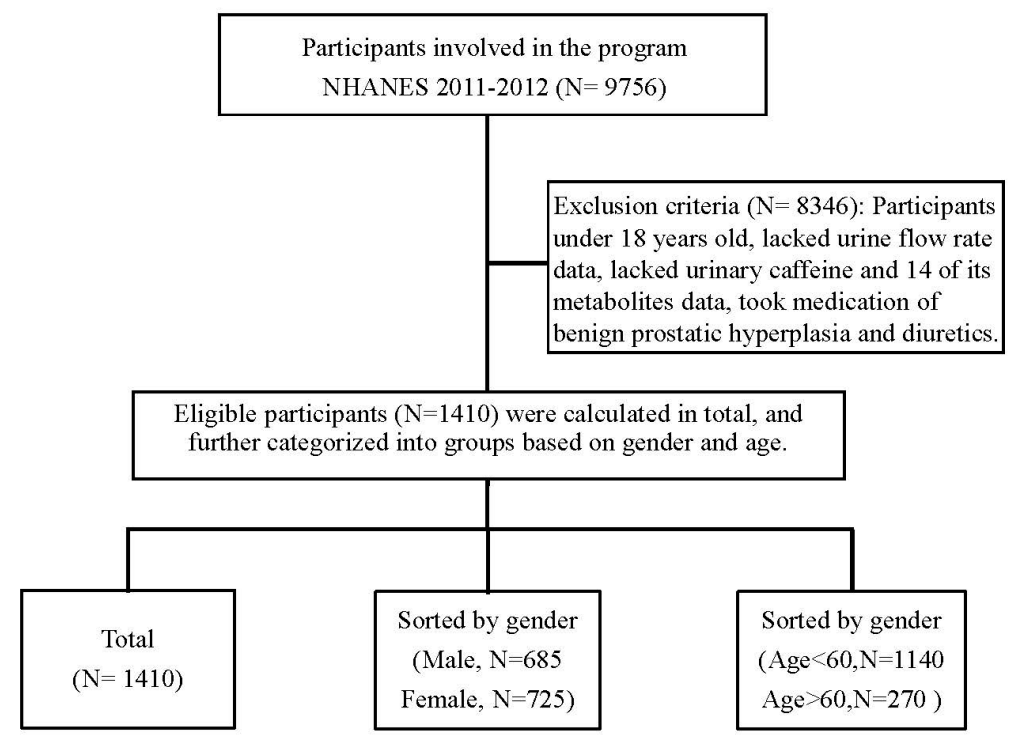

Figure 1. Flow chart of participant recruitment.

Caffeine and 14 of its urinary metabolites, 15 in total, were examined, including 1-methyluric acid, 3-methyluric acid, 7-methyluric acid, 1,3-dimethyluric acid, 1,7-dimethyluric acid, 3,7-dimethyluric acid, 1,3,7-trimethyluric acid, 1-methylxanthine, 3-methylxanthine, 7-methylxanthine, 1,3-dimethylxanthine (theophylline), 1,7-dimethylxanthine (paraxanthine), 3,7-dimethylxanthine (theobromine), 1,3,7-trimethylxanthine (caffeine), and 5-acetylamino-6-amino-3-methyluracil (AAMU). AAMU is the decomposition product of the relatively unstable caffeine metabolite 5-acetylamino-6-formylamino-3-methyluracil (AFMU). Samples were allowed to incubate for at least $30 \mathrm{~min}$ at room temperature so that conversion of all AFMU to the more stable AAMU was ensured.

The lower limit of detection (LLOD in umol/L) for caffeine and caffeine metabolites can be obtained from the NHANES website. For analytes with results below the lower limit of detection, the value is the lower limit of detection divided by the square root of 2 (LLOD/sqrt [2]). All presented data satisfied quality control $(\mathrm{QC})$ procedures, which were performed by a multirule quality control system. Samples examined were collected from 3 QC pools (low-, medium-, and high-quality control pools). Urine analyte concentrations were adjusted to urinary creatinine $(\mathrm{uCr})$ by dividing urine concentration of metabolites by $\mathrm{uCr}$ values.

\subsection{Measurement of Urine Flow Rate}

Urinary flow rate was not a regular examined item in every cycle of NHANES. We collected our data from NHANES 2011-2012. Upon visiting MECs, participants reported the time of the last urinary void at home. At the center, the urinary volume was measured, and the time of sample collection was recorded. Up to three voids could be collected if the initial two voiding volumes were insufficient for the clinical and laboratory analyses. Conceptually, the calculation of urine flow rate is by dividing the volume of the present urine sample by the time duration between the former urination and the present urine collection, i.e., (total urine volume)/(total time duration).

\subsection{Covariates}

The self-reported demographic details of all subjects comprise gender, age, race/ethnicity, smoking history, and medical history. Race was sorted into groups including Mexican American, other Hispanic, non-Hispanic whites and blacks, and other races. Both former and current smokers were defined as having a habit of smoking. The formula of body mass index (BMI) is weight in kilograms divided by height in meters squared $\left(\mathrm{kg} / \mathrm{m}^{2}\right)$. Heart disease was defined as ever been diagnosed with congestive heart failure, coronary heart disease, angina, or heart attack. Biochemical data are measured as follows: 
aspartate aminotransferase (AST) were detected by the Beckman Coulter UniCel DxC 800 Synchron Clinical System; fasting plasma glucose (FPG) levels and urine creatinine (Cr) were measured by Roche/Hitachi Modular P Chemistry Analyzer. Further details about collection procedures are available on the NHANES website.

\subsection{Statistical Analysis}

We conducted a statistical analysis using SPSS (IBM SPSS Statistics for Windows, version 22.0, released 2013; IBM Corp., Armonk, NY). Qualitative data and quantitative variables were reported in percentages and medians and interquartile ranges (IQRs), respectively. A $p$-value of $\leq 0.05$ was considered statistically significant. The urine flow rates deviated from normality, and, thus, log-transformation was performed to achieve normalization. Subsequently, we applied linear regression models to investigate the relationship between urine levels of caffeine metabolite levels and the log-transformed urine flow rate.

Four models were provided in each analytic group to adjust for relevant covariates. The unadjusted model was numbered Model 1; Model 2 was adjusted for age, gender, and race; Model 3 was further adjusted for BMI, serum fasting glucose, AST, and urine creatinine; Model 4 was further adjusted for experiences of heart disease, smoking status, water intake, and caffeine intake.

\section{Results}

\subsection{Characteristics of the Study Population}

The demographic information of the eligible subjects in the study is shown in Table 1 . The mean age of the participants was $47.7 \pm 17.79$ years old, and $49.8 \%$ of participants were male and $43.5 \%$ were ever-smokers. Median of baseline variables is as follows: BMI $28.89 \mathrm{~kg} / \mathrm{m}^{2}$, AST $25.54 \mathrm{U} / \mathrm{L}$, $\mathrm{uCr} 0.89 \mathrm{mg} / \mathrm{dL}$, and FPG $102.92 \mathrm{mg} / \mathrm{dL}$.

\subsection{Urinary Caffeine Metabolite Concentrations and Urine Flow Rate}

Associations between urinary caffeine metabolite concentrations and urine flow rate are demonstrated in Table 2. Positive correlations were discovered by linear regression analysis in caffeine and several of its metabolites: 1 -methyluric acid ( $\beta$ coefficient $=0.068, p<0.001$ ), 1,7-dimethyluric acid ( $\beta$ coefficient $=0.091, p=0.047), 1,3,7$-trimethyluric acid $(\beta$ coefficient $=1.806, p=0.007)$, 1-methylxanthine $(\beta$ coefficient $=0.152, p<0.001), 7$-methylxanthine $(\beta$ coefficient $=0.07, p=0.005), 1,3$-dimethylxanthine (theophylline, $\beta$ coefficient $=1.177, p<0.001)$, 1,7-dimethylxanthine (paraxanthine, $\beta$ coefficient $=0.587, p<0.001$ ), 3,7-dimethylxanthine (theobromine, $\beta$ coefficient $=0.316, p<0.001$ ), 1,3,7-trimethylxanthine (caffeine, $\beta$ coefficient $=$ $1.102, p<0.001$ ), and 5-acetylamino-6-amino-3-methyluracil ( $\beta$ coefficient $=0.053, p=0.004$ ). Notably, additional adjustments for all covariates did not affect the statistical significance in the aforementioned metabolites. We further categorized our participants into subgroups by gender in Table 3 and by age in Table 4. Paraxanthine ( $p<0.001$ in total population, male, female, under and over 60 years old), theobromine ( $p<0.001$ in total population, male, female, under 60 years old; $p=0.011$ over 60 years old), and caffeine ( $p<0.001$ in total population, male, female, under and over 60 years old) were the three showing significant positive correlations in all subgroups. 
Table 1. Characteristics of participants.

\begin{tabular}{|c|c|}
\hline Variables & Median (IQR) or Percent (\%) \\
\hline \multicolumn{2}{|c|}{ Continuous variables } \\
\hline Age (years) & $47.70 \pm 17.79$ \\
\hline $\mathrm{BMI}\left(\mathrm{kg} / \mathrm{m}^{2}\right)$ & $28.89 \pm 6.85$ \\
\hline Aspartate aminotransferase (AST)(U/L) & $25.54 \pm 14.01$ \\
\hline urine creatinine $(\mathrm{mg} / \mathrm{dL})$ & $0.89 \pm 0.29$ \\
\hline serum fasting glucose (mg/dL) & $102.92 \pm 41.51$ \\
\hline 1-methyluric acid (umol/L) & $0.91 \pm 1.19$ \\
\hline 3-methyluric acid (umol/L) & $0.01 \pm 0.02$ \\
\hline 7-methyluric acid (umol/L) & $0.22 \pm 0.34$ \\
\hline 1,3-dimethyluric acid (umol/L) & $0.11 \pm 0.26$ \\
\hline 1,7-dimethyluric acid (umol/L) & $0.42 \pm 0.49$ \\
\hline 3,7-dimethyluric acid (umol/L) & $0.01 \pm 0.02$ \\
\hline 1,3,7-trimethyluric acid (umol/L) & $0.03 \pm 0.03$ \\
\hline 1-methylxanthine (umol/L) & $0.48 \pm 0.68$ \\
\hline 3-methylxanthine (umol/L) & $0.42 \pm 0.61$ \\
\hline 7-methylxanthine (umol/L) & $0.67 \pm 0.92$ \\
\hline 1,3-dimethylxanthine (theophylline) (umol/L) & $0.03 \pm 0.07$ \\
\hline 1,7-dimethylxanthine (paraxanthine) (umol/L) & $0.29 \pm 0.40$ \\
\hline 3,7-dimethylxanthine (theobromine) (umol/L) & $0.28 \pm 0.44$ \\
\hline 1,3,7-trimethylxanthine (caffeine) (umol/L) & $0.09 \pm 0.18$ \\
\hline 5-acetylamino-6-amino-3-methyluracil (uM/L) & $0.96 \pm 1.25$ \\
\hline Caffeine intake on the exam day (mg) & $142.74 \pm 192.73$ \\
\hline Total plain water drank the day before exam (mg) & $1130.95 \pm 1213.59$ \\
\hline \multicolumn{2}{|c|}{ Categorical variables } \\
\hline \multicolumn{2}{|l|}{ Gender } \\
\hline Male & 49.8 \\
\hline Female & 50.2 \\
\hline \multicolumn{2}{|l|}{ Race } \\
\hline Mexican American & 9.5 \\
\hline Other Hispanic & 10.5 \\
\hline Non-Hispanic White & 36.9 \\
\hline Non-Hispanic Black & 26.8 \\
\hline Other Race-including Multi-Racial & 16.4 \\
\hline \multicolumn{2}{|l|}{ Heart disease-ever had a diagnosis } \\
\hline Congestive heart failure & 3.5 \\
\hline Coronary heart disease & 4 \\
\hline Angina & 2.6 \\
\hline Heart attack & 3.8 \\
\hline Smoking & 43.5 \\
\hline
\end{tabular}


Table 2. Association between urinary caffeine metabolites and urine flow rate.

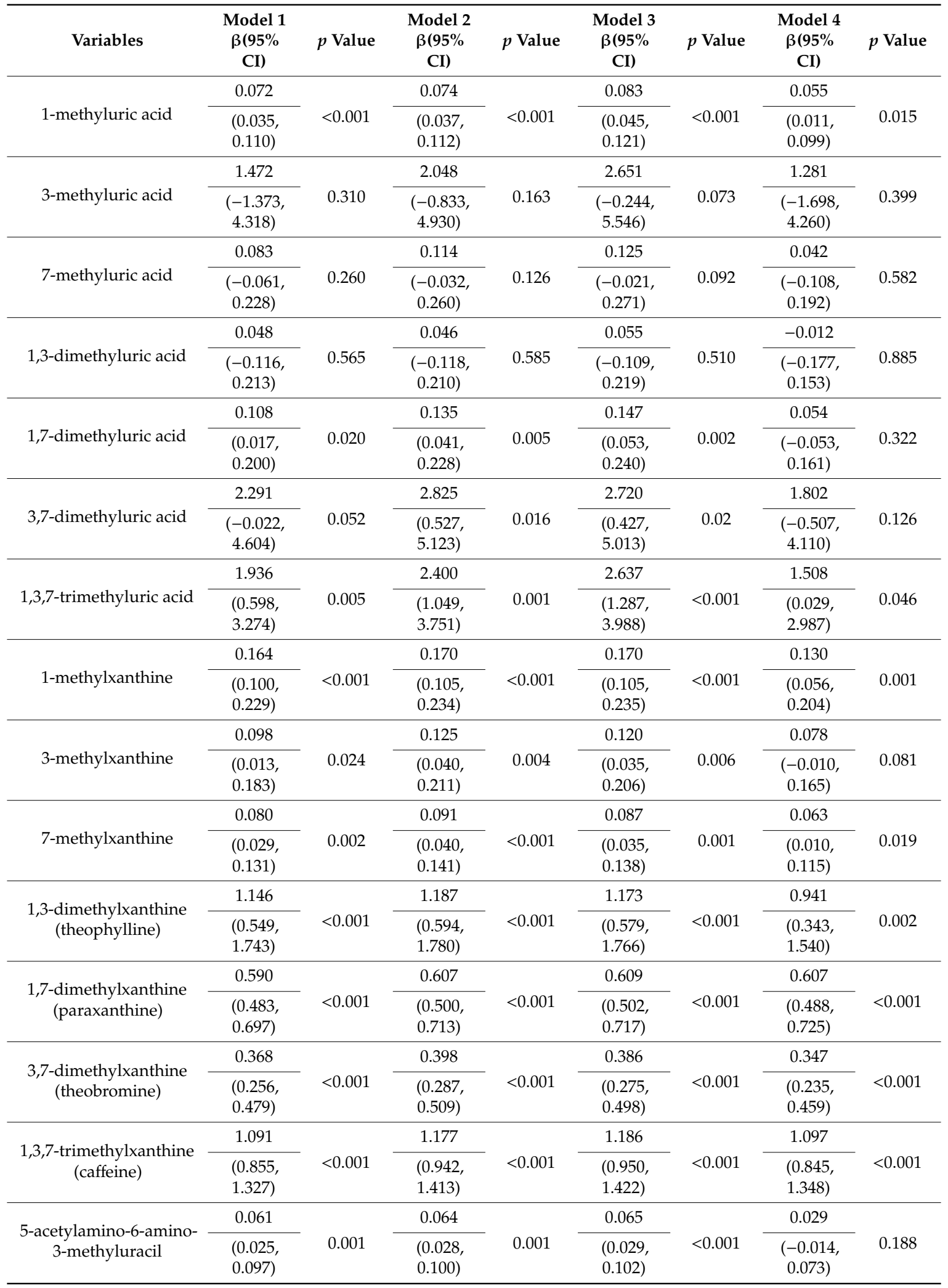

Model 1 = unadjusted. Model 2 = Model $1+$ age, gender, and race/ethnicity. Model $3=$ Model $2+$ BMI, serum fasting glucose, aspartate aminotransferase (AST), and urine creatinine. Model $4=$ Model $3+$ congestive heart failure, coronary heart disease, angina, heart attack, smoking, caffeine intake, and water intake. CI, confidence interval. 
Table 3. Association between urinary caffeine metabolites and urine flow rate as categorized by gender.

\begin{tabular}{|c|c|c|c|c|c|c|c|c|c|}
\hline \multirow{2}{*}{ Variables } & & \multicolumn{4}{|c|}{ Male } & \multicolumn{4}{|c|}{ Female } \\
\hline & & Model 1 & Model 2 & Model 3 & Model 4 & Model 1 & Model 2 & Model 3 & Model 4 \\
\hline \multirow[t]{2}{*}{ 1-methyluric acid } & $\beta(95 \% \mathrm{CI})$ & $\begin{array}{c}0.094 \\
(0.046,0.142)\end{array}$ & $\begin{array}{c}0.099 \\
(0.050,0.148)\end{array}$ & $\begin{array}{c}0.110 \\
(0.061,0.159)\end{array}$ & $\begin{array}{c}0.089 \\
(0.028,0.149)\end{array}$ & $\begin{array}{c}0.051 \\
(-0.006,0.108)\end{array}$ & $\begin{array}{c}0.050 \\
(-0.007,0.108)\end{array}$ & $\begin{array}{c}0.053 \\
(-0.006,0.111)\end{array}$ & $\begin{array}{c}0.026 \\
(-0.039,0.091)\end{array}$ \\
\hline & $p$ value & $<0.001$ & $<0.001$ & $<0.001$ & 0.004 & 0.078 & 0.087 & 0.078 & 0.436 \\
\hline \multirow[t]{2}{*}{ 3-methyluric acid } & $\beta(95 \% \mathrm{CI})$ & $\begin{array}{c}2.721 \\
(-0.861,6.303)\end{array}$ & $\begin{array}{c}2.878 \\
(-0.771,6.526)\end{array}$ & $\begin{array}{c}3.305 \\
(-0.353,6.962)\end{array}$ & $\begin{array}{c}2.007 \\
(-1.785,5.798)\end{array}$ & $\begin{array}{c}1.262 \\
(-3.159,5.683)\end{array}$ & $\begin{array}{c}1.089 \\
(-3.441,5.620)\end{array}$ & $\begin{array}{c}1.492 \\
(-3.083,6.067)\end{array}$ & $\begin{array}{c}-0.043 \\
(-4.770,4.685)\end{array}$ \\
\hline & $p$ value & 0.136 & 0.122 & 0.077 & 0.299 & 0.575 & 0.637 & 0.522 & 0.986 \\
\hline \multirow[t]{2}{*}{ 7-methyluric acid } & $\beta(95 \% \mathrm{CI})$ & $\begin{array}{c}0.203 \\
(-0.005,0.412) \\
\end{array}$ & $\begin{array}{c}0.214 \\
(0.002,0.426) \\
\end{array}$ & $\begin{array}{c}0.231 \\
(0.020,0.443) \\
\end{array}$ & $\begin{array}{c}0.130 \\
(-0.094,0.354) \\
\end{array}$ & $\begin{array}{c}0.052 \\
(-0.147,0.252) \\
\end{array}$ & $\begin{array}{c}0.045 \\
(-0.158,0.249) \\
\end{array}$ & $\begin{array}{c}0.043 \\
(-0.160,0.246) \\
\end{array}$ & $\begin{array}{c}-0.024 \\
(-0.236,0.187) \\
\end{array}$ \\
\hline & $p$ value & 0.056 & 0.048 & 0.032 & 0.256 & 0.607 & 0.661 & 0.677 & 0.823 \\
\hline \multirow{2}{*}{ 1,3-dimethyluric acid } & $\beta(95 \% \mathrm{CI})$ & $\begin{array}{c}0.012 \\
(-0.145,0.170)\end{array}$ & $\begin{array}{c}0.013 \\
(-0.145,0.171)\end{array}$ & $\begin{array}{c}0.021 \\
(-0.136,0.179)\end{array}$ & $\begin{array}{c}0.015 \\
(-0.173,0.142)\end{array}$ & $\begin{array}{c}0.433 \\
(-0.175,1.042)\end{array}$ & $\begin{array}{c}0.428 \\
(-0.195,1.052)\end{array}$ & $\begin{array}{c}0.468 \\
(-0.160,1.096)\end{array}$ & $\begin{array}{c}0.039 \\
(-0.679,0.758)\end{array}$ \\
\hline & $p$ value & 0.876 & 0.869 & 0.789 & 0.847 & 0.163 & 0.178 & 0.144 & 0.915 \\
\hline \multirow[t]{2}{*}{ 1,7-dimethyluric acid } & $\beta(95 \% \mathrm{CI})$ & $\begin{array}{c}0.254 \\
(0.129,0.378) \\
\end{array}$ & $\begin{array}{c}0.271 \\
(0.143,0.400) \\
\end{array}$ & $\begin{array}{c}0.281 \\
(0.153,0.409) \\
\end{array}$ & $\begin{array}{c}0.205 \\
(0.051,0.359) \\
\end{array}$ & $\begin{array}{c}0.032 \\
(-0.100,0.163) \\
\end{array}$ & $\begin{array}{c}0.027 \\
(-0.108,0.162) \\
\end{array}$ & $\begin{array}{c}0.032 \\
(-0.103,0.167) \\
\end{array}$ & $\begin{array}{c}-0.061 \\
(-0.212,0.091) \\
\end{array}$ \\
\hline & $p$ value & $<0.001$ & $<0.001$ & $<0.001$ & 0.009 & 0.637 & 0.695 & 0.644 & 0.433 \\
\hline \multirow[t]{2}{*}{ 3,7-dimethyluric acid } & $\beta(95 \% \mathrm{CI})$ & $\begin{array}{c}3.537 \\
(0.185,6.889)\end{array}$ & $\begin{array}{c}3.551 \\
(0.192,6.910)\end{array}$ & $\begin{array}{c}3.487 \\
(0.139,6.836)\end{array}$ & $\begin{array}{c}2.580 \\
(-0.812,5.973)\end{array}$ & $\begin{array}{c}2.357 \\
(-0.829,5.542)\end{array}$ & $\begin{array}{c}2.377 \\
(-0.814,5.568)\end{array}$ & $\begin{array}{c}2.123 \\
(-1.061,5.308)\end{array}$ & $\begin{array}{c}1.214 \\
(-2.026,4.453)\end{array}$ \\
\hline & $p$ value & 0.039 & 0.038 & 0.041 & 0.136 & 0.147 & 0.144 & 0.191 & 0.462 \\
\hline \multirow[t]{2}{*}{ 1,3,7-trimethyluric acid } & $\beta(95 \% \mathrm{CI})$ & $\begin{array}{c}3.807 \\
(1.895,5.720) \\
\end{array}$ & $\begin{array}{c}4.005 \\
(2.046,5.965) \\
\end{array}$ & $\begin{array}{c}4.325 \\
(2.365,6.284) \\
\end{array}$ & $\begin{array}{c}3.194 \\
(0.958,5.431) \\
\end{array}$ & $\begin{array}{c}1.367 \\
(-0.496,3.229) \\
\end{array}$ & $\begin{array}{c}1.340 \\
(-0.542,3.222) \\
\end{array}$ & $\begin{array}{c}1.439 \\
(-0.445,3.323) \\
\end{array}$ & $\begin{array}{c}0.431 \\
(-1.559,2.482) \\
\end{array}$ \\
\hline & $p$ value & $<0.001$ & $<0.001$ & $<0.001$ & 0.005 & 0.150 & 0.162 & 0.134 & 0.654 \\
\hline \multirow{2}{*}{ 1-methylxanthine } & $\beta(95 \% \mathrm{CI})$ & $\begin{array}{c}0.218 \\
(0.133,0.303)\end{array}$ & $\begin{array}{c}0.221 \\
(0.135,0.306)\end{array}$ & $\begin{array}{c}0.223 \\
(0.137,0.309)\end{array}$ & $\begin{array}{c}0.190 \\
(0.088,0.292)\end{array}$ & $\begin{array}{c}0.125 \\
(0.030,0.221)\end{array}$ & $\begin{array}{c}0.125 \\
(0.029,0.221)\end{array}$ & $\begin{array}{c}0.119 \\
(0.022,0.217)\end{array}$ & $\begin{array}{c}0.088 \\
(-0.018,0.194)\end{array}$ \\
\hline & $p$ value & $<0.001$ & $<0.001$ & $<0.001$ & $<0.001$ & 0.010 & 0.011 & 0.017 & 0.103 \\
\hline \multirow[t]{2}{*}{ 3-methylxanthine } & $\beta(95 \% \mathrm{CI})$ & $\begin{array}{c}0.169 \\
(0.048,0.290)\end{array}$ & $\begin{array}{c}0.173 \\
(0.051,0.295)\end{array}$ & $\begin{array}{c}0.170 \\
(0.051,0.295)\end{array}$ & $\begin{array}{c}0.123 \\
(-0.002,0.249)\end{array}$ & $\begin{array}{c}0.093 \\
(-0.026,0.213)\end{array}$ & $\begin{array}{c}0.092 \\
(-0.029,0.212)\end{array}$ & $\begin{array}{c}0.081 \\
(-0.040,0.202)\end{array}$ & $\begin{array}{c}0.042 \\
(-0.082,0.167)\end{array}$ \\
\hline & $p$ value & 0.006 & 0.006 & 0.007 & 0.054 & 0.126 & 0.135 & 0.191 & 0.503 \\
\hline
\end{tabular}


Table 3. Cont.

\begin{tabular}{|c|c|c|c|c|c|c|c|c|c|}
\hline \multirow{2}{*}{ Variables } & & \multicolumn{4}{|c|}{ Male } & \multicolumn{4}{|c|}{ Female } \\
\hline & & Model 1 & Model 2 & Model 3 & Model 4 & Model 1 & Model 2 & Model 3 & Model 4 \\
\hline \multirow{2}{*}{ 7-methylxanthine } & $\beta(95 \% \mathrm{CI})$ & $\begin{array}{c}0.129 \\
(0.052,0.205)\end{array}$ & $\begin{array}{c}0.129 \\
(0.052,0.206) \\
\end{array}$ & $\begin{array}{c}0.129 \\
(0.052,0.207)\end{array}$ & $\begin{array}{c}0.101 \\
(0.021,0.182)\end{array}$ & $\begin{array}{c}0.068 \\
(0.000,0.137) \\
\end{array}$ & $\begin{array}{c}0.069 \\
(0.000,0.137) \\
\end{array}$ & $\begin{array}{c}0.060 \\
(-0.009,0.130)\end{array}$ & $\begin{array}{c}0.043 \\
(-0.029,0.115)\end{array}$ \\
\hline & $p$ value & 0.001 & 0.001 & 0.001 & 0.013 & 0.050 & 0.050 & 0.088 & 0.238 \\
\hline \multirow{2}{*}{$\begin{array}{l}\text { 1,3-dimethylxanthine } \\
\text { (theophylline) }\end{array}$} & $\beta(95 \% \mathrm{CI})$ & $\begin{array}{c}0.515 \\
(-0.072,1.101)\end{array}$ & $\begin{array}{c}0.519 \\
(-0.070,1.109)\end{array}$ & $\begin{array}{c}0.530 \\
(-0.057,1.118)\end{array}$ & $\begin{array}{c}0.403 \\
(-0.182,0.988)\end{array}$ & $\begin{array}{c}5.657 \\
(3.490,7.373)\end{array}$ & $\begin{array}{c}5.696 \\
(3.967,7.425)\end{array}$ & $\begin{array}{c}5.681 \\
(3.922,7.440)\end{array}$ & $\begin{array}{c}5.309 \\
(3.376,7.242)\end{array}$ \\
\hline & $p$ value & 0.085 & 0.084 & 0.077 & 0.177 & $<0.001$ & $<0.001$ & $<0.001$ & $<0.001$ \\
\hline \multirow{2}{*}{$\begin{array}{l}\text { 1,7-dimethylxanthine } \\
\text { (paraxanthine) }\end{array}$} & $\beta(95 \% \mathrm{CI})$ & $\begin{array}{c}0.596 \\
(0.460,0.732)\end{array}$ & $\begin{array}{c}0.607 \\
(0.470,0.744) \\
\end{array}$ & $\begin{array}{c}0.602 \\
(0.465,0.740)\end{array}$ & $\begin{array}{c}0.607 \\
(0.453,0.760)\end{array}$ & $\begin{array}{c}0.609 \\
(0.446,0.773)\end{array}$ & $\begin{array}{c}0.609 \\
(0.445,0.774)\end{array}$ & $\begin{array}{c}0.610 \\
(0.442,0.777)\end{array}$ & $\begin{array}{c}0.605 \\
(0.421,0.789)\end{array}$ \\
\hline & $p$ value & $<0.001$ & $<0.001$ & $<0.001$ & $<0.001$ & $<0.001$ & $<0.001$ & $<0.001$ & $<0.001$ \\
\hline \multirow{2}{*}{$\begin{array}{l}\text { 3,7-dimethylxanthine } \\
\text { (theobromine) }\end{array}$} & $\beta(95 \% \mathrm{CI})$ & $\begin{array}{c}0.436 \\
(0.279,0.593)\end{array}$ & $\begin{array}{c}0.439 \\
(0.282,0.597)\end{array}$ & $\begin{array}{c}0.425 \\
(0.267,0.583) \\
\end{array}$ & $\begin{array}{c}0.409 \\
(0.249,0.568) \\
\end{array}$ & $\begin{array}{c}0.370 \\
(0.213,0.527) \\
\end{array}$ & $\begin{array}{c}0.371 \\
(0.214,0.529) \\
\end{array}$ & $\begin{array}{c}0.356 \\
(0.198,0.514)\end{array}$ & $\begin{array}{c}0.308 \\
(0.147,0.468) \\
\end{array}$ \\
\hline & $p$ value & $<0.001$ & $<0.001$ & $<0.001$ & $<0.001$ & $<0.001$ & $<0.001$ & $<0.001$ & $<0.001$ \\
\hline \multirow[t]{2}{*}{ 1,3,7-trimethylxanthine (caffeine) } & $\beta(95 \% \mathrm{CI})$ & $\begin{array}{c}1.496 \\
(1.136,1.856) \\
\end{array}$ & $\begin{array}{c}1.526 \\
(1.163,1.890) \\
\end{array}$ & $\begin{array}{c}1.514 \\
(1.152,1.876) \\
\end{array}$ & $\begin{array}{c}1.429 \\
(1.033,1.826) \\
\end{array}$ & $\begin{array}{c}0.983 \\
(0.670,1.297) \\
\end{array}$ & $\begin{array}{c}0.989 \\
(0.672,1.306) \\
\end{array}$ & $\begin{array}{c}0.988 \\
(0.670,1.307) \\
\end{array}$ & $\begin{array}{c}0.890 \\
(0.552,1.227) \\
\end{array}$ \\
\hline & $p$ value & $<0.001$ & $<0.001$ & $<0.001$ & $<0.001$ & $<0.001$ & $<0.001$ & $<0.001$ & $<0.001$ \\
\hline \multirow{2}{*}{$\begin{array}{l}\text { 5-acetylamino-6-amino- } \\
\text { 3-methyluracil }\end{array}$} & $\beta(95 \% \mathrm{CI})$ & $\begin{array}{c}0.094 \\
(0.051,0.138)\end{array}$ & $\begin{array}{c}0.098 \\
(0.054,0.143)\end{array}$ & $\begin{array}{c}0.097 \\
(0.052,0.141)\end{array}$ & $\begin{array}{c}0.074 \\
(0.019,0.129)\end{array}$ & $\begin{array}{c}0.023 \\
(-0.034,0.080)\end{array}$ & $\begin{array}{c}0.022 \\
(-0.037,0.080)\end{array}$ & $\begin{array}{c}0.023 \\
(-0.037,0.082)\end{array}$ & $\begin{array}{c}-0.025 \\
(-0.095,0.044)\end{array}$ \\
\hline & $p$ value & $<0.001$ & $<0.001$ & $<0.001$ & 0.008 & 0.429 & 0.468 & 0.454 & 0.472 \\
\hline
\end{tabular}

Model 1 = unadjusted. Model 2 = Model $1+$ age, gender, and race/ethnicity. Model 3 = Model $2+$ BMI, serum fasting glucose, aspartate aminotransferase (AST), and urine creatinine.

Model 4 = Model $3+$ congestive heart failure, coronary heart disease, angina, heart attack, smoking, caffeine intake, and water intake. 
Table 4. Association between urinary caffeine metabolites and urine flow rate as categorized by age.

\begin{tabular}{|c|c|c|c|c|c|c|c|c|c|}
\hline \multirow{2}{*}{ Variables } & & \multicolumn{4}{|c|}{ Age $<60$} & \multicolumn{4}{|c|}{ Age $\geq 60$} \\
\hline & & Model 1 & Model 2 & Model 3 & Model 4 & Model 1 & Model 2 & Model 3 & Model 4 \\
\hline \multirow{2}{*}{ 1-methyluric acid } & $\beta(95 \% \mathrm{CI})$ & $\begin{array}{c}0.102 \\
(0.0538,0.146) \\
\end{array}$ & $\begin{array}{c}0.098 \\
(0.053,0.142) \\
\end{array}$ & $\begin{array}{c}0.103 \\
(0.058,0.148) \\
\end{array}$ & $\begin{array}{c}0.076 \\
(0.025,0.127) \\
\end{array}$ & $\begin{array}{c}0.004 \\
(-0.068,0.075) \\
\end{array}$ & $\begin{array}{c}0.001 \\
(-0.070,0.072) \\
\end{array}$ & $\begin{array}{c}0.006 \\
(-0.066,0.079) \\
\end{array}$ & $\begin{array}{c}-0.003 \\
(-0.126,0.060) \\
\end{array}$ \\
\hline & $p$ value & $<0.001$ & $<0.001$ & $<0.001$ & 0.004 & 0.920 & 0.987 & 0.861 & 0.483 \\
\hline \multirow{2}{*}{ 3-methyluric acid } & $\beta(95 \% \mathrm{CI})$ & $\begin{array}{c}3.936 \\
(0.409,7.462)\end{array}$ & $\begin{array}{c}3.833 \\
(0.299,7.368)\end{array}$ & $\begin{array}{c}4.190 \\
(0.641,7.740)\end{array}$ & $\begin{array}{c}2.595 \\
(-1.129,6.318)\end{array}$ & $\begin{array}{c}-2.656 \\
(-7.598,2.286)\end{array}$ & $\begin{array}{c}-1.480 \\
(-6.431,3.471)\end{array}$ & $\begin{array}{c}-1.003 \\
(-6.017,4.011)\end{array}$ & $\begin{array}{c}-1.733 \\
(-7.035,3.569)\end{array}$ \\
\hline & $p$ value & 0.029 & 0.034 & 0.021 & 0.172 & 0.291 & 0.557 & 0.694 & 0.521 \\
\hline \multirow[t]{2}{*}{ 7-methyluric acid } & $\beta(95 \% \mathrm{CI})$ & $\begin{array}{c}0.212 \\
(0.033,0.391)\end{array}$ & $\begin{array}{c}0.212 \\
(0.033,0.391)\end{array}$ & $\begin{array}{c}0.214 \\
(0.035,0.394)\end{array}$ & $\begin{array}{c}0.130 \\
(-0.057,0.011)\end{array}$ & $\begin{array}{c}-0.134 \\
(-0.384,0.115)\end{array}$ & $\begin{array}{c}-0.063 \\
(-0.313,0.187)\end{array}$ & $\begin{array}{c}-0.065 \\
(-0.315,0.185)\end{array}$ & $\begin{array}{c}-0.156 \\
(-0.423,0.112)\end{array}$ \\
\hline & $p$ value & 0.021 & 0.021 & 0.019 & 0.172 & 0.290 & 0.619 & 0.609 & 0.253 \\
\hline \multirow[t]{2}{*}{ 1,3-dimethyluric acid } & $\beta(95 \% \mathrm{CI})$ & $\begin{array}{c}0.912 \\
(0.428,1.395) \\
\end{array}$ & $\begin{array}{c}0.894 \\
(0.401,1.387) \\
\end{array}$ & $\begin{array}{c}0.943 \\
(0.447,1.438) \\
\end{array}$ & $\begin{array}{c}0.546 \\
(-0.022,1.114)\end{array}$ & $\begin{array}{c}-0.056 \\
(-0.233,0.121)\end{array}$ & $\begin{array}{c}-0.080 \\
(-0.256,0.095)\end{array}$ & $\begin{array}{c}-0.086 \\
(-0.261,0.089)\end{array}$ & $\begin{array}{c}-0.100 \\
(-0.276,0.077) \\
\end{array}$ \\
\hline & $p$ value & $<0.001$ & 0.001 & $<0.001$ & 0.059 & 0.536 & 0.369 & 0.333 & 0.268 \\
\hline \multirow{2}{*}{ 1,7-dimethyluric acid } & $\beta(95 \% \mathrm{CI})$ & $\begin{array}{c}0.220 \\
(0.104,0.337)\end{array}$ & $\begin{array}{c}0.224 \\
(0.105,0.344)\end{array}$ & $\begin{array}{c}0.229 \\
(0.110,0.349)\end{array}$ & $\begin{array}{c}0.132 \\
(-0.003,0.267)\end{array}$ & $\begin{array}{c}-0.056 \\
(-0.206,0.095)\end{array}$ & $\begin{array}{c}-0.045 \\
(-0.196,0.106)\end{array}$ & $\begin{array}{c}-0.044 \\
(-0.196,0.109)\end{array}$ & $\begin{array}{c}-0.124 \\
(-0.305,0.057)\end{array}$ \\
\hline & $p$ value & $<0.001$ & $<0.001$ & $<0.001$ & 0.056 & 0.466 & 0.554 & 0.572 & 0.179 \\
\hline \multirow[t]{2}{*}{ 3,7-dimethyluric acid } & $\beta(95 \% \mathrm{CI})$ & $\begin{array}{c}2.883 \\
(0.280,5.485) \\
\end{array}$ & $\begin{array}{c}3.333 \\
(0.754,5.912) \\
\end{array}$ & $\begin{array}{c}3.317 \\
(0.736,5.897) \\
\end{array}$ & $\begin{array}{c}2.314 \\
(-0.299,4.926) \\
\end{array}$ & $\begin{array}{c}-0.044 \\
(-5.104,5.015) \\
\end{array}$ & $\begin{array}{c}-0.319 \\
(-4.697,5.335) \\
\end{array}$ & $\begin{array}{c}-0.350 \\
(-5.344,4.644) \\
\end{array}$ & $\begin{array}{c}-1.263 \\
(-6.403,3.877) \\
\end{array}$ \\
\hline & $p$ value & 0.030 & 0.011 & 0.012 & 0.083 & 0.986 & 0.900 & 0.890 & 0.629 \\
\hline \multirow[t]{2}{*}{ 1,3,7-trimethyluric acid } & $\beta(95 \% \mathrm{CI})$ & $\begin{array}{c}2.977 \\
(1.365,4.589) \\
\end{array}$ & $\begin{array}{c}3.220 \\
(1.587,4.853) \\
\end{array}$ & $\begin{array}{c}3.366 \\
(1.729,5.002) \\
\end{array}$ & $\begin{array}{c}2.180 \\
(0.420,3.939) \\
\end{array}$ & $\begin{array}{c}-0.146 \\
(-2.565,2.273) \\
\end{array}$ & $\begin{array}{c}-0.058 \\
(-2.482,2.366) \\
\end{array}$ & $\begin{array}{c}0.294 \\
(-2.149,2.737) \\
\end{array}$ & $\begin{array}{c}-0.487 \\
(-3.270,2.297) \\
\end{array}$ \\
\hline & $p$ value & $<0.001$ & $<0.001$ & $<0.001$ & 0.015 & 0.906 & 0.962 & 0.813 & 0.731 \\
\hline \multirow[t]{2}{*}{ 1-methylxanthine } & $\beta(95 \% \mathrm{CI})$ & $\begin{array}{c}0.164 \\
(0.094,0.233) \\
\end{array}$ & $\begin{array}{c}0.160 \\
(0.090,0.229) \\
\end{array}$ & $\begin{array}{c}0.164 \\
(0.093,0.235) \\
\end{array}$ & $\begin{array}{c}0.122 \\
(0.043,0.201) \\
\end{array}$ & $\begin{array}{c}0.162 \\
(-0.012,0.337) \\
\end{array}$ & $\begin{array}{c}0.144 \\
(-0.030,0.317) \\
\end{array}$ & $\begin{array}{c}0.108 \\
(-0.069,0.285)\end{array}$ & $\begin{array}{c}0.068 \\
(-0.146,0.283)\end{array}$ \\
\hline & $p$ value & $<0.001$ & $<0.001$ & $<0.001$ & 0.002 & 0.068 & 0.105 & 0.232 & 0.531 \\
\hline \multirow[t]{2}{*}{ 3-methylxanthine } & $\beta(95 \% \mathrm{CI})$ & $\begin{array}{c}0.142 \\
(0.039,0.244)\end{array}$ & $\begin{array}{c}0.159 \\
(0.056,0.262)\end{array}$ & $\begin{array}{c}0.159 \\
(0.056,0.263) \\
\end{array}$ & $\begin{array}{c}0.105 \\
(-0.001,0.0211)\end{array}$ & $\begin{array}{c}0.009 \\
(-0.146,0.164)\end{array}$ & $\begin{array}{c}0.036 \\
(-0.118,0.191)\end{array}$ & $\begin{array}{c}0.004 \\
(-0.151,0.159)\end{array}$ & $\begin{array}{c}-0.015 \\
(-0.175,0.145) \\
\end{array}$ \\
\hline & $p$ value & 0.007 & 0.002 & 0.003 & 0.051 & 0.908 & 0.643 & 0.957 & 0.855 \\
\hline
\end{tabular}


Table 4. Cont.

\begin{tabular}{|c|c|c|c|c|c|c|c|c|c|}
\hline \multirow{2}{*}{ Variables } & & \multicolumn{4}{|c|}{ Age $<60$} & \multicolumn{4}{|c|}{ Age $\geq 60$} \\
\hline & & Model 1 & Model 2 & Model 3 & Model 4 & Model 1 & Model 2 & Model 3 & Model 4 \\
\hline \multirow{2}{*}{ 7-methylxanthine } & $\beta(95 \% \mathrm{CI})$ & $\begin{array}{c}0.086 \\
(0.029,0.143) \\
\end{array}$ & $\begin{array}{c}0.094 \\
(0.038,0.150) \\
\end{array}$ & $\begin{array}{c}0.093 \\
(0.036,0.150) \\
\end{array}$ & $\begin{array}{c}0.068 \\
(0.010,0.127) \\
\end{array}$ & $\begin{array}{c}0.054 \\
(-0.062,0.170) \\
\end{array}$ & $\begin{array}{c}0.066 \\
(-0.049,0.181) \\
\end{array}$ & $\begin{array}{c}0.035 \\
(-0.081,0.152) \\
\end{array}$ & $\begin{array}{c}0.013 \\
(-0.110,0.135) \\
\end{array}$ \\
\hline & $p$ value & 0.003 & 0.001 & 0.001 & 0.022 & 0.361 & 0.258 & 0.549 & 0.839 \\
\hline \multirow{2}{*}{$\begin{array}{l}\text { 1,3-dimethylxanthine } \\
\text { (theophylline) }\end{array}$} & $\beta(95 \% \mathrm{CI})$ & $\begin{array}{c}5.900 \\
(4.433,7.367) \\
\end{array}$ & $\begin{array}{c}6.267 \\
(4.792,7.742) \\
\end{array}$ & $\begin{array}{c}6.401 \\
(4.911,7.891) \\
\end{array}$ & $\begin{array}{c}5.823 \\
(4.204,7.442) \\
\end{array}$ & $\begin{array}{c}0.283 \\
(-0.378,0.943) \\
\end{array}$ & $\begin{array}{c}0.185 \\
(-0.470,0.841) \\
\end{array}$ & $\begin{array}{c}0.110 \\
(-0.544,0.764) \\
\end{array}$ & $\begin{array}{c}0.059 \\
(-0.602,0.721) \\
\end{array}$ \\
\hline & $p$ value & $<0.001$ & $<0.001$ & $<0.001$ & $<0.001$ & 0.401 & 0.578 & 0.741 & 0.860 \\
\hline \multirow{2}{*}{$\begin{array}{l}\text { 1,7-dimethylxanthine } \\
\text { (paraxanthine) }\end{array}$} & $\beta(95 \% \mathrm{CI})$ & $\begin{array}{c}0.618 \\
(0.487,0.749)\end{array}$ & $\begin{array}{c}0.627 \\
(0.496,0.759)\end{array}$ & $\begin{array}{c}0.640 \\
(0.508,0.773)\end{array}$ & $\begin{array}{c}0.613 \\
(0.469,0.757)\end{array}$ & $\begin{array}{c}0.536 \\
(0.349,0.724)\end{array}$ & $\begin{array}{c}0.513 \\
(0.326,0.700)\end{array}$ & $\begin{array}{c}0.479 \\
(0.287,0.671) \\
\end{array}$ & $\begin{array}{c}0.532 \\
(0.314,0.750)\end{array}$ \\
\hline & $p$ value & $<0.001$ & $<0.001$ & $<0.001$ & $<0.001$ & $<0.001$ & $<0.001$ & $<0.001$ & $<0.001$ \\
\hline \multirow{2}{*}{$\begin{array}{l}\text { 3,7-dimethylxanthine } \\
\text { (theobromine) }\end{array}$} & $\beta(95 \% \mathrm{CI})$ & $\begin{array}{c}0.376 \\
(0.245,0.507)\end{array}$ & $\begin{array}{c}0.407 \\
(0.278,0.537)\end{array}$ & $\begin{array}{c}0.405 \\
(0.275,0.536)\end{array}$ & $\begin{array}{c}0.358 \\
(0.227,0.488)\end{array}$ & $\begin{array}{c}0.340 \\
(0.123,0.557)\end{array}$ & $\begin{array}{c}0.344 \\
(0.130,0.559)\end{array}$ & $\begin{array}{c}0.289 \\
(0.071,0.507)\end{array}$ & $\begin{array}{c}0.260 \\
(0.036,0.484)\end{array}$ \\
\hline & $p$ value & $<0.001$ & $<0.001$ & $<0.001$ & $<0.001$ & 0.002 & 0.002 & 0.009 & 0.023 \\
\hline \multirow{2}{*}{$\begin{array}{l}\text { 1,3,7-trimethylxanthine } \\
\text { (caffeine) }\end{array}$} & $\beta(95 \% \mathrm{CI})$ & $\begin{array}{c}1.319 \\
(1.020,1.618)\end{array}$ & $\begin{array}{c}1.377 \\
(1.078,1.676)\end{array}$ & $\begin{array}{c}1.398 \\
(1.097,1.698)\end{array}$ & $\begin{array}{c}1.265 \\
(0.948,1.582)\end{array}$ & $\begin{array}{c}0.763 \\
(0.374,1.152)\end{array}$ & $\begin{array}{c}0.791 \\
(0.405,1.176) \\
\end{array}$ & $\begin{array}{c}0.749 \\
(0.361,1.137)\end{array}$ & $\begin{array}{c}0.762 \\
(0.338,1.186) \\
\end{array}$ \\
\hline & $p$ value & $<0.001$ & $<0.001$ & $<0.001$ & $<0.001$ & $<0.001$ & $<0.001$ & $<0.001$ & $<0.001$ \\
\hline $\begin{array}{l}\text { 5-acetylamino-6-amino- } \\
\text { 3-methyluracil }\end{array}$ & $\begin{array}{c}\beta(95 \% \mathrm{CI}) \\
p \text { value }\end{array}$ & $\begin{array}{c}0.094 \\
(0.048,0.141) \\
<0.001\end{array}$ & $\begin{array}{c}0.088 \\
(0.040,0.135) \\
<0.001\end{array}$ & $\begin{array}{c}0.088 \\
(0.040,0.136) \\
<0.001\end{array}$ & $\begin{array}{c}0.045 \\
(-0.001,0.102) \\
0.117\end{array}$ & $\begin{array}{c}0.020 \\
(-0.037,0.077) \\
0.486\end{array}$ & $\begin{array}{c}0.018 \\
(-0.039,0.074) \\
0.539\end{array}$ & $\begin{array}{c}0.016 \\
(-0.041,0.072) \\
0.591\end{array}$ & $\begin{array}{c}-0.002 \\
(-0.073,0.068) \\
0.946\end{array}$ \\
\hline
\end{tabular}

Model 1 = unadjusted. Model 2 = Model $1+$ age, gender, and race/ethnicity. Model 3 = Model $2+$ BMI, serum fasting glucose, aspartate aminotransferase (AST), and urine creatinine Model 4 = Model $3+$ congestive heart failure, coronary heart disease, angina, heart attack, smoking, caffeine intake, and water intake. 
Table 3 shows the results for male and female subgroups, respectively. In the male subgroup, caffeine and 14 of its metabolites revealed positive correlations with urine flow rate, in contrast with the female subgroup, with a number of 5. Among them, 1-methyluric acid, 7-methyluric acid, 1,7-dimethyluric acid, 3,7-dimethyluric acid, 1,3,7-trimethyluric acid, 3-methylxanthine, 7-methylxanthine, and 5-acetylamino-6-amino-3-methyluracil presented significance in males but not females. 1,3-Dimethylxanthine (theophylline) was the only one showing a positive correlation in females but marginal significance in males ( $p$-value $=0.067$ in males, $p$-value $<0.001$ in females).

Table 4 shows the results in participants aged under and over 60 years old, respectively, referring to the cutoff point of the elderly population agreed on by the United Nations [20]. In the subgroup of age $<60$ years old, caffeine and 14 of its metabolites all revealed positive correlations. On the contrary, in the elderly subgroup $\geq 60$ years old, the number of metabolites presenting significant correlations shrank to 3 , namely, paraxanthine ( $p$-value $<0.001)$, theobromine ( $p$-value $=0.011)$, and caffeine ( $p$-value $<0.001)$.

\section{Discussion}

In the US population, we found that urine levels of caffeine metabolites were positively associated with urine flow rate. Furthermore, there are more caffeine metabolites showing a flow-dependency in males than females and more in younger participants than older ones. Notably, caffeine and two of its metabolites, paraxanthine and theobromine, revealed significance across all subgroups. They were the main primary metabolites of caffeine, with paraxanthine composing $84 \%$ and theobromine composing $12 \%$ [18]. Other metabolites were formed by successive demethylations and hydroxylations. The large proportion of caffeine metabolites being flow-dependent across subgroups proves that urine flow rate is a nonnegligible influencing factor in caffeine excretion.

Previous literature discussing caffeine metabolites and urine flow rate focused more on theophylline, which not only belongs to the same methylxanthine family as caffeine but is itself also one of the main urinary metabolites $[16,17,21-25]$. Previous studies have mentioned the renal clearance of theophylline being highly dependent on urine flow rate $[16,17,21-23]$. Some pharmacologists have developed a mathematical model that explains the dependence of renal clearance on urine flow rate in drugs such as theophylline, ethanol, and butabarbital [24,25]. Being siblings in the same methylxanthine family, with similar metabolic pathways, caffeine and 14 of its metabolites presenting flow-dependency in our study is plausible.

Other studies focusing directly on caffeine were of limited sample size and did not mention comparisons among gender and age, two unneglectable factors that determine urine flow rate. One early study observed a positive association in 10 elderly men [26], while another proposed a positive relationship in 16 volunteers [27]. Several reports mentioned different flow-dependency of caffeine metabolite ratios (MRs) contributing to different roles in the assessment of cytochrome P450 1A2 (CYP1A2) activity $[28,29]$. These studies revealed evident results but lacked further comparisons in the subgroups. Our study was composed of 1410 participants from a representative sample, providing highly robust evidence with a much bigger number of participants, and made comparisons in subgroups to investigate whether different tendencies existed.

In the present study, we observed differences in the number of caffeine metabolites showing a relationship with urine flow rate. Males outnumbered females, and younger participants outnumbered older ones. We surveyed the factors affecting caffeine metabolism and urine flow rate and found possible reasons for this difference. The rate of caffeine metabolism primarily depends on the genetic variability of enzymes dominating caffeine breakdown, smoking status, alcohol intake, specific medications, liver diseases, and pregnancy [30-32]. CYPIA2, the main enzyme metabolizing caffeine, is known to show higher activity in men [33]. In contrast, female hormones decrease CYPIA2 activity during pregnancy and with oral contraceptive use [34]. Furthermore, studies have described that males show a significant decline in urinary flow rate with age, whereas females show less variation in urine flow rate with respect to age [35]. In sum, a slower rate of caffeine metabolism and a more constant feature of urine flow rate in females may explain the observation of less metabolites being flow-dependent 
in this group. In addition, the urine flow rate declines with age due to various reasons aside from the one that was already excluded in our study, benign prostate hyperplasia. Prolapsed bladder after vaginal childbirth and menopause in females are also factors that may influence urine flow rate [35]. In the elderly group, increased factors affecting the urine flow rate may perturb and weaken the flow-dependency of metabolites.

The potential mechanism that links urinary caffeine metabolites and the urine flow rate together might contribute to the physiological interplay in the kidneys. Tang-Liu et al. established a model that explained drugs with a dependence of renal clearance on urine flow rate [24]. Theophylline and caffeine fall into the category of those that are reabsorbed in the renal tubule, but the diffusional rate is less than that of water. This results in a disequilibrium state that causes the renal clearance of these drugs to be dependent on urine flow rates. Other drugs that showed no flow-dependency were either not reabsorbed at all or their diffusional rate was equal to or greater than that of water. In another study, the rate-concentration curve of theophylline was depicted to be convex-ascending but not linear. The clearance of drugs increases markedly with urine flow up to a certain degree and, thereafter, increases only slightly [18].

The clinical application of assessing caffeine and its metabolites in urine is multifaceted. Medical research fields extend physiology, psychology, and pharmacology. The finding in our study illustrates the necessity of controlling urine flow rates in studies relevant to caffeine, especially when subjects are male and with younger participants. Restriction of fluid intake, salt intake, avoidance of diuretic foods, and adjustment of renal function are reasonable means to approach more adequate assessment. Moreover, other practice could be inferred from this observation. Increasing urine flow rate can act as a means of detoxifying in caffeine-overdosed patients. Those who wish to stay awake by caffeine may consider avoiding factors that speed up their urine flow rate. These practices require further studies to validate their effectiveness. However, they are worth a try according to the flow-dependent feature demonstrated in this study.

Several limitations of this study should be mentioned. Firstly, NHANES is a cross-sectional study in which urinary caffeine metabolites and urine flow rate were examined at one particular time point rather than continuously collected for a long period of time. The causal relationship could not be established due to possible biased results by single measurement. Secondly, we put more emphasis on the dataset from NHANES 2011-2012 but less on NHANES 2009-2010, which was another cycle that collected urinary caffeine metabolite data. The official website mentioned the different instruments used in the two cycles, which may cause analytical bias due to the earlier one requiring the specimens to be analyzed twice. Even so, the results of 2009-2010 of 1853 participants in Table A1 still revealed a similar trend of flow-dependency, only that the amount of metabolites is not the same. Thirdly, we measured the average urine flow rate rather than the peak urine flow rate. Combining both certainly offers a more comprehensive view of urodynamic studies, but peak flow rate requires more complicated calculations with uroflowmetry. Previous comparable studies have utilized the average urine flow rate, as shown in Table 5 [17,21-23,25-29], and thus we followed their practice. 
Table 5. Summary of the literature review findings on the association between urinary caffeine metabolites and urine flow rate.

\begin{tabular}{|c|c|c|c|c|c|}
\hline Study Details & Study Design & Participants & $\begin{array}{c}\text { Caffeine } \\
\text { Metabolites }\end{array}$ & $\begin{array}{c}\text { Evaluation of } \\
\text { Urine Flow } \\
\text { Rate } \\
\end{array}$ & $\begin{array}{c}\text { Findings on Urinary } \\
\text { Caffeine Metabolites and } \\
\text { Urine Flow Rate }\end{array}$ \\
\hline \multicolumn{6}{|c|}{ Caffeine } \\
\hline Our study & $\begin{array}{l}\text { cross-sectional } \\
\text { study }\end{array}$ & $\mathrm{N}=1410$ & $\begin{array}{l}\text { Caffeine and } 14 \text { of its } \\
\text { metabolites }\end{array}$ & $\begin{array}{l}\text { Average flow } \\
\text { rate }\end{array}$ & $\begin{array}{l}\text { Positive correlations were } \\
\text { shown between several } \\
\text { urinary metabolites and urine } \\
\text { flow rate. Men showed more } \\
\text { correlation than females, and } \\
\text { the young (age }<60 \text { ) showed } \\
\text { more correlation than the } \\
\text { elderly (age }>60) \text {. }\end{array}$ \\
\hline $\begin{array}{l}\text { Blanchard, J. } \\
\text { et al. (1983), } \\
\text { Scotland [25] }\end{array}$ & $\begin{array}{l}\text { cross-sectional } \\
\text { study }\end{array}$ & $N=16$ & Caffeine & $\begin{array}{l}\text { Average flow } \\
\text { rate }\end{array}$ & $\begin{array}{l}\text { Positive correlation between } \\
\text { the renal clearance of both } \\
\text { unbound (CLU) and total } \\
\text { (CLR) caffeine and the mean } \\
\text { urine flow rate. }\end{array}$ \\
\hline $\begin{array}{l}\text { Trang, J.M. et al. } \\
\text { (1985), USA } \\
\text { [24] }\end{array}$ & $\begin{array}{l}\text { cross-sectional } \\
\text { study }\end{array}$ & $\mathrm{N}=10$ & Caffeine & $\begin{array}{l}\text { Average flow } \\
\text { rate }\end{array}$ & $\begin{array}{l}\text { Positive correlations were } \\
\text { observed between total body } \\
\text { clearance (CL), renal clearance } \\
\text { (CL), and nonrenal clearance } \\
\text { (CL) and urine flow rate (UFR) }\end{array}$ \\
\hline $\begin{array}{c}\text { Sinués, B. et al. } \\
\text { (1999), Spain } \\
\text { [26] }\end{array}$ & $\begin{array}{l}\text { cross-sectional } \\
\text { study }\end{array}$ & $\mathrm{N}=125$ & $\begin{array}{l}5 \text { urinary caffeine } \\
\text { metabolite ratios } \\
\text { (MRs) }\end{array}$ & $\begin{array}{l}\text { Average flow } \\
\text { rate }\end{array}$ & $\begin{array}{l}\text { MR1, MR3, and MR4 were the } \\
\text { most flow-dependent. MR2 } \\
\text { was flow-independent. MR5 } \\
\text { was less flow-dependent. }\end{array}$ \\
\hline $\begin{array}{l}\text { Sinués, B. et al. } \\
\text { (2002), Spain } \\
\text { [27] }\end{array}$ & $\begin{array}{l}\text { cross-sectional } \\
\text { study }\end{array}$ & $\mathrm{N}=152$ & $\begin{array}{l}8 \text { caffeine metabolites } \\
\text { and } 5 \text { urinary } \\
\text { caffeine metabolite } \\
\text { ratios (MRs) }\end{array}$ & $\begin{array}{l}\text { Average flow } \\
\text { rate }\end{array}$ & $\begin{array}{c}7 \text { caffeine metabolites were } \\
\text { flow-dependent. MR1, MR3, } \\
\text { and MR4 were } \\
\text { flow-dependent. MR2 and } \\
\text { MR5 were flow-independent. }\end{array}$ \\
\hline \multicolumn{6}{|c|}{ Theophylline } \\
\hline Our study & $\begin{array}{l}\text { cross-sectional } \\
\text { study }\end{array}$ & $N=1410$ & Theophylline & $\begin{array}{l}\text { Average flow } \\
\text { rate }\end{array}$ & $\begin{array}{l}\text { Positive correlations were } \\
\text { shown between theophylline } \\
\text { and urine flow rate in the } \\
\text { female subgroup and the } \\
\text { young (age <60) subgroup. }\end{array}$ \\
\hline $\begin{array}{l}\text { Gerhard Levy. } \\
\text { et al. (1976), } \\
\text { USA [17] }\end{array}$ & $\begin{array}{l}\text { cross-sectional } \\
\text { study }\end{array}$ & $N=6$ & Theophylline & $\begin{array}{l}\text { Average flow } \\
\text { rate }\end{array}$ & $\begin{array}{c}\text { Positive correlation was } \\
\text { shown between the renal } \\
\text { clearance of theophylline and } \\
\text { the urine flow rate. }\end{array}$ \\
\hline $\begin{array}{l}\text { Tang-Liu, } \\
\text { D.D.S. et al. } \\
\text { (1982), USA } \\
\text { [23] }\end{array}$ & $\begin{array}{l}\text { cross-sectional } \\
\text { study }\end{array}$ & $\mathrm{N}=14$ & Theophylline & $\begin{array}{l}\text { Average flow } \\
\text { rate }\end{array}$ & $\begin{array}{l}\text { Theophylline renal clearance } \\
\text { is highly dependent on urine } \\
\text { flow rate and is neither } \\
\text { concentration- nor } \\
\text { dose-related. }\end{array}$ \\
\hline $\begin{array}{l}\text { St-Pierre, M.V. } \\
\text { et al. (1985), } \\
\text { USA [19] }\end{array}$ & $\begin{array}{l}\text { cross-sectional } \\
\text { study }\end{array}$ & $\mathrm{N}=8$ & $\begin{array}{l}\text { Theophylline and } 3 \\
\text { of its major } \\
\text { metabolites }\end{array}$ & $\begin{array}{l}\text { Average flow } \\
\text { rate }\end{array}$ & $\begin{array}{l}\text { Renal clearance of metabolites } \\
\text { was greater after morning } \\
\text { dosing, the time with } \\
\text { enhanced urine flow rate. }\end{array}$ \\
\hline $\begin{array}{l}\text { Bonnacker, I. et } \\
\text { al. (1989), } \\
\text { Germany [20] }\end{array}$ & $\begin{array}{l}\text { cross-sectional } \\
\text { study }\end{array}$ & $\mathrm{N}=10$ & $\begin{array}{l}\text { Theophylline and } 3 \\
\text { of its metabolites }\end{array}$ & $\begin{array}{l}\text { Average flow } \\
\text { rate }\end{array}$ & $\begin{array}{l}\text { The renal clearance of } \\
\text { 1,3-DMU, the main metabolite } \\
\text { of theophylline, was found to } \\
\text { depend both upon urine flow } \\
\text { rate and age. }\end{array}$ \\
\hline $\begin{array}{l}\text { Agbaba, D. et } \\
\text { al., (1990), } \\
\text { Yugoslavia [21] }\end{array}$ & $\begin{array}{l}\text { cross-sectional } \\
\text { study }\end{array}$ & $N=22$ & Theophylline & $\begin{array}{l}\text { Average flow } \\
\text { rate }\end{array}$ & $\begin{array}{l}\text { The dependence of the renal } \\
\text { excretion of theophylline on } \\
\text { urine flow rate was found } \\
\text { after both IV administration } \\
\text { and at steady state. }\end{array}$ \\
\hline
\end{tabular}




\section{Conclusions}

A positive association exists between several urinary caffeine metabolites and urine flow rate. The number of metabolites showing certain flow-dependency is higher in males than females and also higher in young participants compared to elderly participants. Further studies are necessary to elucidate the mechanisms underlying the flow-dependency appearance of caffeine metabolites in urine. Our study highlights the importance of considering the urine flow rate as an influencing factor in interpretations of urinary data regarding caffeine.

Author Contributions: Conceptualization, S.E.W. and W.-L.C.; methodology, W.-L.C.; software, W.-L.C.; validation, S.E.W. and W.-L.C.; formal analysis, S.E.W. and W.-L.C.; investigation, S.E.W. and W.-L.C.; resources, W.-L.C.; data curation, S.E.W. and W.-L.C.; writing-original draft preparation, S.E.W.; writing-review and editing, S.E.W. and W.-L.C.; visualization, S.E.W. and W.-L.C.; supervision, W.-L.C. All authors have read and agreed to the published version of the manuscript.

Funding: This research received no external funding.

Conflicts of Interest: The authors declare no conflict of interest.

\section{Appendix A}

Table A1. Association between urinary caffeine metabolites and urine flow rate in NHANES 2009-2010.

\begin{tabular}{|c|c|c|c|c|c|c|c|c|}
\hline Variables & $\begin{array}{c}\text { Model } 1 \\
\beta(95 \% \\
\text { CI })\end{array}$ & $p$ Value & $\begin{array}{c}\text { Model } 2 \\
\beta(95 \% \\
\text { CI })\end{array}$ & $p$ Value & $\begin{array}{c}\text { Model } 3 \\
\beta(95 \% \\
\text { CI })\end{array}$ & $p$ Value & $\begin{array}{c}\text { Model } 4 \\
\beta(95 \% \\
\text { CI })\end{array}$ & $p$ Value \\
\hline \multirow[b]{2}{*}{ 1-methyluric acid } & 0.041 & \multirow[b]{2}{*}{0.008} & 0.049 & \multirow[b]{2}{*}{0.001} & 0.050 & \multirow[b]{2}{*}{0.001} & 0.032 & \multirow[b]{2}{*}{0.072} \\
\hline & $\begin{array}{l}(0.011, \\
0.070)\end{array}$ & & $\begin{array}{l}\text { (0.019, } \\
0.079)\end{array}$ & & $\begin{array}{l}(0.020, \\
0.080)\end{array}$ & & $\begin{array}{c}(-0.003, \\
0.066)\end{array}$ & \\
\hline \multirow[b]{2}{*}{ 3-methyluric acid } & -1.087 & \multirow[b]{2}{*}{0.307} & 0.096 & \multirow[b]{2}{*}{0.930} & 0.826 & \multirow[b]{2}{*}{0.073} & 1.281 & \multirow[b]{2}{*}{0.399} \\
\hline & $\begin{array}{c}(-3.174 \\
1.000)\end{array}$ & & $\begin{array}{c}(-2.045 \\
2.236)\end{array}$ & & $\begin{array}{c}(-0.244 \\
3.027)\end{array}$ & & $\begin{array}{c}(-1.698 \\
2.282)\end{array}$ & \\
\hline \multirow[b]{2}{*}{ 7-methyluric acid } & -0.002 & \multirow[b]{2}{*}{0.968} & 0.061 & \multirow[b]{2}{*}{0.287} & 0.072 & \multirow[b]{2}{*}{0.215} & 0.025 & \multirow[b]{2}{*}{0.670} \\
\hline & $\begin{array}{c}(-0.112 \\
0.228)\end{array}$ & & $\begin{array}{c}(-0.052 \\
0.260)\end{array}$ & & $\begin{array}{c}(-0.042 \\
0.271)\end{array}$ & & $\begin{array}{c}(-2.157 \\
0.192)\end{array}$ & \\
\hline \multirow[b]{2}{*}{ 1,3-dimethyluric acid } & 0.006 & \multirow[b]{2}{*}{0.851} & 0.015 & \multirow[b]{2}{*}{0.626} & 0.014 & \multirow[b]{2}{*}{0.630} & 0.008 & \multirow[b]{2}{*}{0.783} \\
\hline & $\begin{array}{c}(-0.064 \\
0.065)\end{array}$ & & $\begin{array}{c}(-0.045 \\
0.074)\end{array}$ & & $\begin{array}{c}(-0.046 \\
0.073)\end{array}$ & & $\begin{array}{c}(-0.051, \\
0.067)\end{array}$ & \\
\hline \multirow[b]{2}{*}{ 1,7-dimethyluric acid } & 0.097 & \multirow[b]{2}{*}{0.008} & 0.136 & \multirow[b]{2}{*}{$<0.001$} & 0.137 & \multirow[b]{2}{*}{$<0.001$} & 0.088 & \multirow[b]{2}{*}{0.031} \\
\hline & $\begin{array}{l}(0.025, \\
0.169)\end{array}$ & & $\begin{array}{l}(0.064, \\
0.209)\end{array}$ & & $\begin{array}{l}(0.064, \\
0.209)\end{array}$ & & $\begin{array}{l}(0.008, \\
0.167)\end{array}$ & \\
\hline \multirow[b]{2}{*}{ 3,7-dimethyluric acid } & 0.561 & \multirow[b]{2}{*}{0.557} & 1.367 & \multirow[b]{2}{*}{0.154} & 1.323 & \multirow[b]{2}{*}{0.169} & 0.714 & \multirow[b]{2}{*}{0.462} \\
\hline & $\begin{array}{c}(-1.314 \\
2.435)\end{array}$ & & $\begin{array}{c}(-0.515 \\
3.249)\end{array}$ & & $\begin{array}{c}(-0.561 \\
3.207)\end{array}$ & & $\begin{array}{c}(-1.191 \\
2.618)\end{array}$ & \\
\hline \multirow{2}{*}{ 1,3,7-trimethyluric acid } & 2.345 & \multirow[b]{2}{*}{$<0.001$} & 2.759 & \multirow[b]{2}{*}{$<0.001$} & 2.770 & \multirow[b]{2}{*}{$<0.001$} & 2.373 & \\
\hline & $\begin{array}{l}\text { (1.405, } \\
3.285)\end{array}$ & & $\begin{array}{l}(1.819, \\
3.698)\end{array}$ & & $\begin{array}{l}(1.832, \\
3.709)\end{array}$ & & $\begin{array}{l}1.383 \\
3.363)\end{array}$ & $<0.001$ \\
\hline & 0.124 & & 0.131 & & 0.127 & & 0.101 & \\
\hline 1-methylxanthine & $\begin{array}{l}(0.067, \\
0.180)\end{array}$ & $<0.001$ & $\begin{array}{l}0.075, \\
0.187)\end{array}$ & $<0.001$ & $\begin{array}{l}(0.071, \\
0.184)\end{array}$ & $<0.001$ & $\begin{array}{l}(0.037 \\
0.166)\end{array}$ & 0.002 \\
\hline & 0.022 & & 0.064 & & 0.063 & & 0.038 & \\
\hline 3-methylxanthine & $\begin{array}{c}(-0.043 \\
0.090)\end{array}$ & 0.526 & $\begin{array}{c}(-0.005 \\
0.132)\end{array}$ & 0.071 & $\begin{array}{c}(-0.006 \\
0.132)\end{array}$ & 0.073 & $\begin{array}{c}(-0.016 \\
0.108)\end{array}$ & 0.280 \\
\hline & 0.037 & & 0.052 & & 0.050 & & 0.032 & \\
\hline 7-methylxanthine & $\begin{array}{c}(-0.010 \\
0.083)\end{array}$ & 0.125 & $\begin{array}{l}(0.006, \\
0.099)\end{array}$ & 0.028 & $\begin{array}{l}(0.003, \\
0.097)\end{array}$ & 0.037 & $\begin{array}{l}(0.010 \\
0.081)\end{array}$ & 0.189 \\
\hline
\end{tabular}


Table A1. Cont.

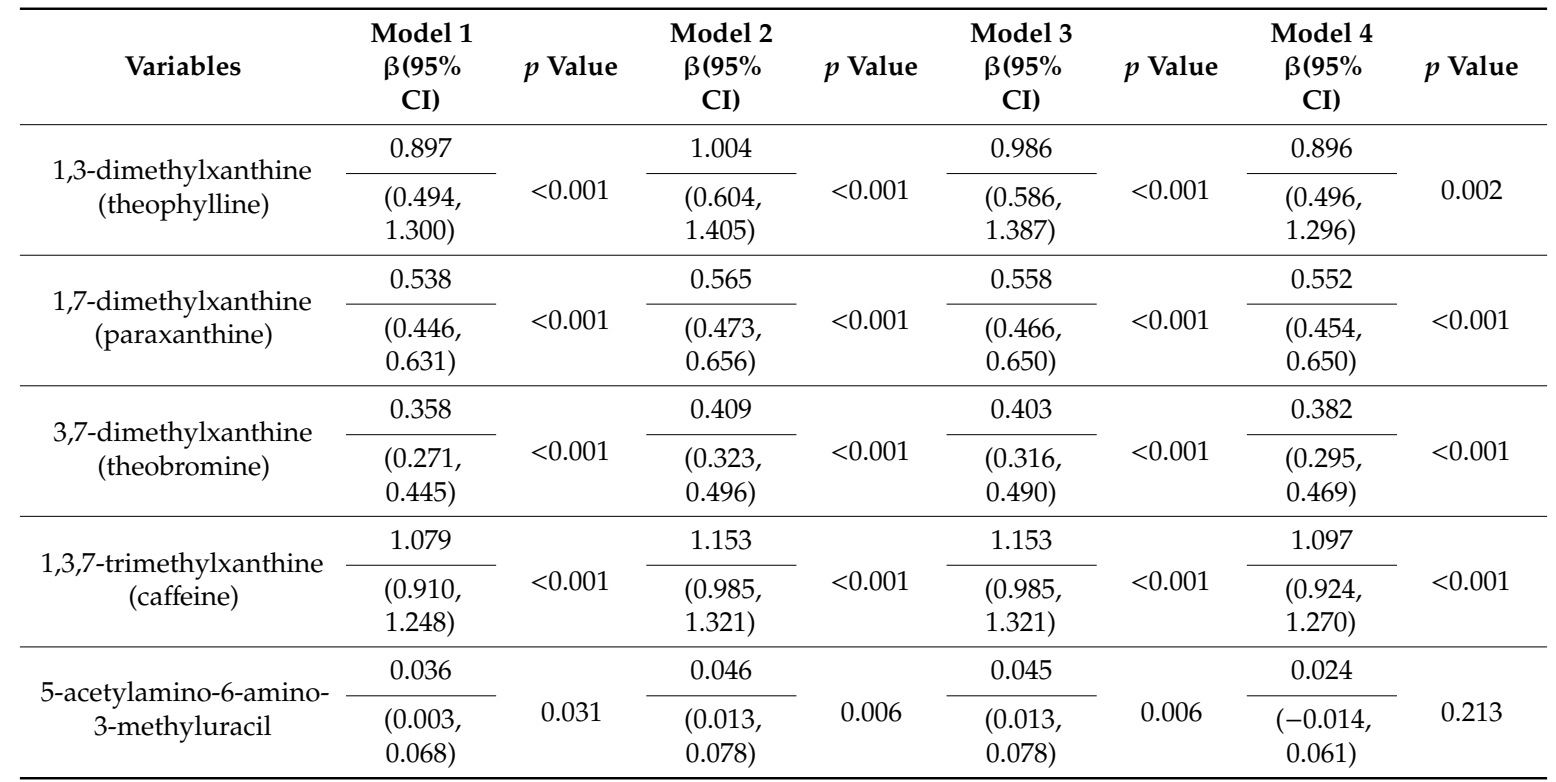

Model 1 = unadjusted. Model 2 = Model $1+$ age, gender, and race/ethnicity. Model 3 = Model $2+$ BMI, serum fasting glucose, aspartate aminotransferase (AST), and urine creatinine. Model $4=$ Model $3+$ congestive heart failure, coronary heart disease, angina, heart attack, smoking, caffeine intake, and water intake.

\section{References}

1. Nehlig, A.; Daval, J.L.; Debry, G. Caffeine and the central nervous system: Mechanisms of action, biochemical, metabolic and psychostimulant effects. Brain Res. Brain Res. Rev. 1992, 17, 139-170. [CrossRef]

2. Sattin, A.; Rall, T.W. The effect of adenosine and adenine nucleotides on the cyclic adenosine $3^{\prime}, 5^{\prime}$-phosphate content of guinea pig cerebral cortex slices. Mol. Pharmacol. 1970, 6, 13-23.

3. Huang, Z.-L.; Qu, W.-M.; Eguchi, N.; Chen, J.-F.; Schwarzschild, M.A.; Fredholm, B.B.; Urade, Y.; Hayaishi, O. Adenosine A 2A, but not A 1, receptors mediate the arousal effect of caffeine. Nat. Neurosci. 2005, 8, 858-859. [CrossRef]

4. Shi, D.; Nikodijević, O.; Jacobson, K.A.; Daly, J.W. Chronic caffeine alters the density of adenosine, adrenergic, cholinergic, GABA, and serotonin receptors and calcium channels in mouse brain. Cell. Mol. Neurobiol. 1993, 13, 247-261. [CrossRef] [PubMed]

5. Nawrot, P.; Jordan, S.; Eastwood, J.; Rotstein, J.; Hugenholtz, A.; Feeley, M. Effects of caffeine on human health. Food Addiv. Contam. 2003, 20,1-30. [CrossRef] [PubMed]

6. Pelchovitz, D.J.; Goldberger, J.J. Caffeine and cardiac arrhythmias: A review of the evidence. Am. J. Med. 2011, 124, 284-289. [CrossRef] [PubMed]

7. Rieg, T.; Steigele, H.; Schnermann, J.; Richter, K.; Osswald, H.; Vallon, V. Requirement of intact adenosine A1 receptors for the diuretic and natriuretic action of the methylxanthines theophylline and caffeine. J. Pharmacol. Exp. Ther. 2005, 313, 403-409. [CrossRef] [PubMed]

8. Ogawa, N.; Ueki, H.J.P.; Neurosciences, C. Clinical importance of caffeine dependence and abuse. Psychiatry Clin. Neurosci. 2007, 61, 263-268. [CrossRef]

9. Roehrs, T.; Roth, T. Caffeine: Sleep and daytime sleepiness. Sleep Med. Rev. 2008, 12, 153-162. [CrossRef]

10. Angelucci, M.E.; Vital, M.A.; Cesário, C.; Zadusky, C.R.; Rosalen, P.L.; Da Cunha, C. The effect of caffeine in animal models of learning and memory. Eur. J. Pharmacol. 1999, 373, 135-140. [CrossRef]

11. Kalow, W.; Tang, B.K. Use of caffeine metabolite ratios to explore CYP1A2 and xanthine oxidase activities. Clin. Pharmacol. Ther. 1991, 50, 508-519. [CrossRef] [PubMed]

12. Butler, M.; Lang, N.; Young, J.; Caporaso, N.; Vineis, P.; Hayes, R.; Teitel, C.; Massengill, J.; Lawsen, M.; Kadlubar, F.J.P. Determination of CYP1A2 and NAT2 phenotypes in human populations by analysis of caffeine urinary metabolites. Pharmacogenetics 1992, 2, 116-127. [CrossRef] [PubMed] 
13. Fujimaki, M.; Saiki, S.; Li, Y.; Kaga, N.; Taka, H.; Hatano, T.; Ishikawa, K.-I.; Oji, Y.; Mori, A.; Okuzumi, A.J.N. Serum caffeine and metabolites are reliable biomarkers of early Parkinson disease. Neurology 2018, 90, e404-e411. [CrossRef] [PubMed]

14. Benowitz, N.L. Clinical pharmacology of caffeine. Ann. Rev. Med. 1990, 41, 277-288. [CrossRef] [PubMed]

15. Faber, M.S.; Jetter, A.; Fuhr, U. Assessment of CYP1A2 activity in clinical practice: Why, how, and when? Basic Clin. Pharmacol. Toxicol. 2005, 97, 125-134. [CrossRef]

16. Birkett, D.; Dahlqvist, R.; Miners, J.; Lelo, A.; Billing, B. Comparison of theophylline and theobromine metabolism in man. Drug Metab. Dispos. 1985, 13, 725-728.

17. Levy, G.; Koysooko, R. Renal clearance of theophylline in man. J. Clin. Pharmacol. 1976, 16, 329-332. [CrossRef]

18. Tang-Liu, D.; Williams, R.; Riegelman, S. Disposition of caffeine and its metabolites in man. J. Pharmacol. Exp. Ther. 1983, 224, 180-185.

19. Roebuck, J. When does old age begin?: The evolution of the English definition. J. Soc. Hist. 1979, 12, 416-428. [CrossRef]

20. United Nations. Department of Economic; Affairs, S. World Population Ageing, 1950-2050; United Nations Publications: New York, NY, USA, 2002.

21. St-Pierre, M.V.; Spino, M.; Isles, A.F.; Tesoro, A.; MacLeod, S.M. Temporal variation in the disposition of theophylline and its metabolites. Clin. Pharmacol. Ther. 1985, 38, 89-95. [CrossRef]

22. Bonnacker, I.; Berdel, D.; Süverkrüp, R.; Berg, A.V. Renal clearance of theophylline and its major metabolites: Age and urine flow dependency in paediatric patients. Eur. J. Clin. Pharmacol. 1989, 36, 145-150. [CrossRef]

23. Agbaba, D.; Pokrajac, M.; Varagić, V.M.; Pešić, V. Dependence of the renal excretion of theophylline on its plasma concentrations and urine flow rate in asthmatic children. J. Pharm. Pharmacol. 1990, 42, 827-830. [CrossRef] [PubMed]

24. Tang-Liu, D.D.-S.; Tozer, T.N.; Riegelman, S. Dependence of renal clearance on urine flow: A mathematical model and its application. J. Pharm. Sci. 1983, 72, 154-158. [CrossRef]

25. Tang-Liu, D.D.-S.; Tozer, T.N.; Riegelman, S. Urine flow-dependence of theophylline renal clearance in man. J. Pharmacokinet. Biopharm. 1982, 10, 351-364. [CrossRef] [PubMed]

26. Trang, J.M.; Blanchard, J.; Conrad, K.A.; Harrison, G.G. Relationship between total body clearance of caffeine and urine flow rate in elderly men. Biopharm. Drug Dispos. 1985, 6, 51-56. [CrossRef]

27. Blanchard, J.; Sawers, S.J.A. Relationship between urine flow rate and renal clearance of caffeine in man. J. Clin. Pharmacol. 1983, 23, 134-138. [CrossRef]

28. Sinués, B.; Sáenz, M.A.; Lanuza, J.; Bernal, M.L.; Fanlo, A.; Juste, J.L.; Mayayo, E. Five caffeine metabolite ratios to measure tobacco-induced CYP1A2 activity and their relationships with urinary mutagenicity and urine flow. Cancer Epidemiol. Prev. Biomark. 1999, 8, 159-166.

29. Sinués, B.; Fanlo, A.; Bernal, M.L.; Mayayo, E.; Soriano, M.A.; Martínez-Ballarin, E. Influence of the urine flow rate on some caffeine metabolite ratios used to assess CYP1A2 activity. Ther. Drug Monit. 2002, 24, 715-721. [CrossRef]

30. Kalow, W.; Tang, B.K. Caffeine as a metabolic probe: Exploration of the enzyme-inducing effect of cigarette smoking. Clin. Pharmacol. Ther. 1991, 49, 44-48. [CrossRef]

31. George, J.; Murphy, T.; Roberts, R.; Cooksley, W.G.; Halliday, J.W.; Powell, L.W. Influence of alcohol and caffeine consumption on caffeine elimination. Clin. Exp. Pharmacol. Physiol. 1986, 13, 731-736. [CrossRef]

32. Carrillo, J.A.; Benitez, J. Clinically significant pharmacokinetic interactions between dietary caffeine and medications. Clin. Pharmacokinet. 2000, 39, 127-153. [CrossRef] [PubMed]

33. Relling, M.V.; Lin, J.S.; Ayers, G.D.; Evans, W.E. Racial and gender differences in N-acetyltransferase, xanthine oxidase, and CYP1A2 activities. Clin. Pharmacol. Ther. 1992, 52, 643-658. [CrossRef] [PubMed]

34. Tracy, T.S.; Venkataramanan, R.; Glover, D.D.; Caritis, S.N. Temporal changes in drug metabolism (CYP1A2, CYP2D6 and CYP3A Activity) during pregnancy. Am. J. Obstet. Gynecol. 2005, 192, 633-639. [CrossRef] [PubMed]

35. Haylen, B.T.; Ashby, D.; Sutherst, J.R.; Frazer, M.I.; West, C.R. Maximum and average urine flow rates in normal male and female populations-the Liverpool nomograms. Br. J. Urol. 1989, 64, 30-38. [CrossRef]

(C) 2020 by the authors. Licensee MDPI, Basel, Switzerland. This article is an open access article distributed under the terms and conditions of the Creative Commons Attribution (CC BY) license (http://creativecommons.org/licenses/by/4.0/). 